\title{
Unrecognized Out of Hospital Cardiac Arrest Symptoms during Thailand's Emergency Medical Services
}

\author{
Thanat Tangpaisarn ${ }^{1}$ D, Tachaya Srinopparatanakul ${ }^{1}$, Ratchanan Artpru $^{2}$, Praew Kotruchin ${ }^{1}$ D, Kamonwon lenghong ${ }^{1}$, \\ Korakot Apiratwarakul $^{1 *}$ (D) \\ ${ }^{1}$ Department of Emergency Medicine, Faculty of Medicine, Khon Kaen University, Khon Kaen, Thailand; ${ }^{2}$ Emergency medical \\ services unit, Srinagarind Hospital, Faculty of Medicine, Khon Kaen University, Khon Kaen, Thailand
}

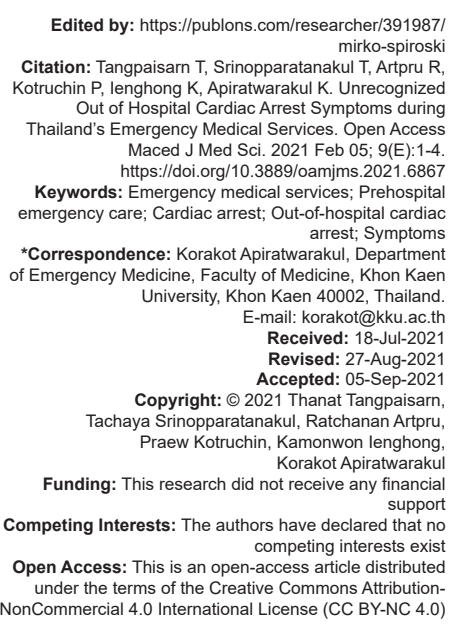

\section{Abstract}

BACKGROUND: To improve survival rate, the main focus of adult cardiac arrest management includes rapid recognition, prompt administration of cardiopulmonary resuscitation (CPR), defibrillation for shockable rhythms, postreturn of spontaneous circulation care, and identification and treatment of underlying causes. This study aimed to identify the determinants of unrecognized cardiac arrest and to study the recognition rate of out-of-hospital cardiac arrest $(\mathrm{OHCA})$ by emergency medical services (EMS) call handlers.

METHODS: We included all OHCA patients who were transferred to hospital via EMS of Srinagarind hospital, Khon Kaen, Thailand, from January 01,2020 , to December 31, 2020. The primary outcome was to identify symptoms that lead to an unrecognized cardiac arrest by the EMS call handlers. Secondary outcomes were to identify the recognition rate of OHCA by EMS call handlers, and assess the outcome of CPR.

RESULTS: There were a total of 58 patients in the present study, 26 patients $(44.8 \%)$ and 32 patients $(55.2 \%)$ belonged to the unrecognized and recognized cardiac arrest groups, respectively. The most common symptoms that led to unrecognized cardiac arrest were a state of unconsciousness (46.2\%), major trauma (15.4\%), and seizurelike activity $(11.5 \%)$. The rate of survival to hospital discharge was higher in the recognized cardiac arrest group $(6.3 \%$ vs. $0 \%)$

CONCLUSIONS: Falling unconscious is the most common symptom of unrecognized OHCA cases seen by EMS in Thailand. Basic life support, especially an immediate assessment of a patient's respiratory status should be taught in health programs in school or through public service channels.

\section{Introduction}

Survival and recovery from adult cardiac arrest depend on the coordinating efforts of a complex system to secure the best outcome for the victim. The main focus in adult cardiac arrest events includes rapid recognition, prompt administration of cardiopulmonary resuscitation (CPR), defibrillation of malignant shockable rhythms, post-return of spontaneous circulation (ROSC) supportive care, and the identification and treatment of underlying causes [1]. Typical survival rates were around $8.8 \%$, which was relatively higher among patients who received bystander CPR [2]. Therefore, fast and accurate recognition of out-of-hospital cardiac arrest $(\mathrm{OHCA})$ by the Emergency Medical Services (EMS) call handlers is essential.

EMS call handlers to have three roles in OHCA: (1) Recognition; (2) facilitation of rapid EMS attendance; and (3) provision of bystander resuscitation instructions including identification of public access defibrillators [3]. In Thailand, the EMS's task is guided by criteria-based dispatch algorithms. However, using these algorithms properly in large part depends on the call handler's skill of interpreting the caller's description of symptoms which has often proven to have been a challenging task [4].

A systematic review of call handler-caller interaction suggested that the most sensitive and specific combination of reported symptoms of OHCA were unconsciousness together with absent or abnormal breathing [5]. However, it is often difficult to obtain an accurate description of the patient's condition and because of these challenges, call handlers' recognition of OHCA ranged from $14.1 \%$ to $96.9 \%$ among studies [6]. These factors can give rise to delayed response, poor rates of bystander resuscitation instructions, and led to reduced survival rates. A recent study has shown that factors such as a reduced or fluctuating level of consciousness, abnormal pulse/ heart rate, and patients being female were associated with a lack of recognition of OHCA by call handlers [3].

Survival to hospital discharge from OHCA in Thailand was about 10\% [7], [8], while survival 
rates in some other countries were reported as up to $25 \%$ [9]. A study in Northern Ireland demonstrated that a contributing factor to low survival rates was poor call handler sensitivity for the identification of OHCA [10]. There are still limited studies about the determinants of unrecognized cardiac arrest, and the recognition rate of OHCA in the Thai population. This study aimed to identify the commonly missed symptoms of those whose cardiac arrest went unrecognized.

\section{Methods}

\section{Study design and population}

This study was conducted in the EMS department of Srinagarind hospital, a 1000-bed tertiary care university hospital located in northeast Thailand, from January 01, 2020, to December 31, 2020.

All OHCA patients who were transferred to Srinagarind hospital via EMS from January 01, 2020, to December 31, 2020, were included in the study. Those who had no dispatched data available were excluded from the study.

This study was conducted in accordance with the principles of the Declaration of Helsinki and Good Clinical Practice guidelines. The Khon Kaen University Ethics Committee for Human Research approved the study and waived the requirement for written individual informed consent (HE641287).

\section{Outcome and statistical analysis}

The primary outcome was to identify symptoms that lead to recognition failure of cardiac arrest by the EMS call handlers. Secondary outcomes were to identify the recognition rate of OHCA by EMS call handlers and the outcome of CPR on OHCA patients in the Northeast of Thailand.

The patient's baseline characteristics and clinical measurements were reported as mean \pm standard deviation (SD) for continuous variables with a normal distribution. Median and minimum - maximum were used to describe continuous variables with non-normal distribution. Number and percentage were used for categorical variables. For comparison between the recognized and unrecognized groups, an independent sample t-test and Mann-Whitney $U$ test were used for continuous variables and Pearson's chi-square or Fisher's exact test for proportions for categorical variables. All statistical analyses were performed using IBM SPSS statistics for Windows, version 27.0 (SPSS Inc).

\section{Results}

We enrolled a total of 58 patients in this study, the total number of patients with unrecognized cardiac arrest was 26 patients (44.8\%), and those with recognized cardiac arrest was 32 patients (55.2\%). Baseline characteristics of patients with unrecognized cardiac arrest differed from those with recognized cardiac arrest in multiple respects, including older age (60 years vs. 50 years, $p=0.019$ ) and fewer bystanders performing CPR prior to the ALS team arrival $(38.5 \%$ vs. $87.5 \%, p<0.001)$. For the outcome of administered CPR, we found that the rate of ROSC was higher in the unrecognized cardiac arrest group (34.6\% vs. $15.6 \%$ ) but none of them survived until hospital discharge. In contrast, even though the rate of ROSC was lower in the recognized cardiac arrest group, some of them survived until hospital discharge (6.3\%) (Table 1).

Table 1: Baseline characteristics of OHCA patients

\begin{tabular}{|c|c|c|c|}
\hline Clinical Characteristics & $\begin{array}{l}\text { Unrecognized cardiac } \\
\text { arrest }(n=26)\end{array}$ & $\begin{array}{l}\text { Recognized cardiac } \\
\text { arrest }(n=32)\end{array}$ & p-value \\
\hline Age (yr) (mean \pm SD) & $60.19 \pm 13.68$ & $50.38 \pm 16.64$ & 0.019 \\
\hline Male, $\mathrm{n}(\%)$ & $21(80.8)$ & $24(75.0)$ & 0.754 \\
\hline Shift, n (\%) & & & 0.755 \\
\hline Morning & $12(46.2)$ & $12(37.5)$ & \\
\hline Afternoon & $9(34.6)$ & $14(43.8)$ & \\
\hline Night & $5(19.2)$ & $6(18.8)$ & \\
\hline Median response time (min-max) & $9.5 \min (6.0-25.0)$ & $9.0 \min (4.0-29.0)$ & 0.672 \\
\hline Median scene time (min-max) & $5.0 \min (2.0-22.0)$ & $5.0 \min (2.0-18.0)$ & 0.956 \\
\hline Initial rhythm, n (\%) & & & 0.209 \\
\hline Asystole/PEA & $25(96.2)$ & $27(84.4)$ & \\
\hline VT/VF & $1(3.8)$ & $5(15.6)$ & \\
\hline AED, n (\%) & $0(0)$ & $0(0)$ & NA \\
\hline $\begin{array}{l}\text { Bystander performing CPR before } \\
\text { ALS team arrival }{ }^{*}, n(\%)\end{array}$ & $10(38.5)$ & $28(87.5)$ & $<0.001$ \\
\hline ROSC, n (\%) & $9(34.6)$ & $5(15.6)$ & 0.126 \\
\hline Survival to discharge, $\mathrm{n}(\%)$ & $0(0)$ & $2(6.3)$ & 0.497 \\
\hline
\end{tabular}

The most common reported symptoms of the unrecognized cardiac arrest were unconsciousness $(46.2 \%)$, followed by the experience of major trauma $(15.4 \%)$ and seizure-like activity $(11.5 \%)$ subsequently. The symptoms that the call handler never missed cardiac arrest were drowning, unconscious with no breathing, and electrical shock (Table 2).

Table 2: Dispatched symptoms

\begin{tabular}{lll}
\hline Symptoms & $\begin{array}{l}\text { Unrecognized cardiac } \\
\text { arrest } \mathrm{n}=26(\%)\end{array}$ & $\begin{array}{l}\text { Recognized cardiac } \\
\text { arrest } \mathrm{n}=32(\%)\end{array}$ \\
\hline Unconscious & $12(46.2)$ & $18(56.3)$ \\
Major trauma & $4(15.4)$ & $6(18.8)$ \\
Seizure-like activity & $3(11.5)$ & $0(0)$ \\
Reduced consciousness & $2(7.7)$ & $1(3.1)$ \\
Falling & $1(3.8)$ & $0(0)$ \\
Malaise/fatigue & $1(3.8)$ & $0(0)$ \\
Noisy breathing & $1(3.8)$ & $0(0)$ \\
Gun shot & $1(3.8)$ & $0(0)$ \\
Hanging & $1(3.8)$ & $1(3.1)$ \\
Drowning & $0(0)$ & $2(6.3)$ \\
Unconscious with no breathing & $0(0)$ & $2(6.3)$ \\
Electrical shock & $0(0)$ & $2(6.3)$ \\
\hline
\end{tabular}

\section{Discussion}

In the present study, we found that the recognition rate of cardiac arrest by call handlers was 
$55.2 \%$, which was in the range of the recent international systematic review (14.1-96.9\%) and similar to the recent Korean study (45.8\%) [6], [11]. However, this recognition rate is relatively low compared to the median recognition rate of cardiac arrest from the international systematic review (73.9\%) [6]. This may reflect the lack of knowledge about basic life support in the Thai laymen population. We also found that a significant factor which affected the recognition of cardiac arrest was the patient's age, the older patients tended to have unrecognized cardiac arrest. Our finding was similar to Viereck et al. [6]. The possible explanation of the finding could be the sedentary lifestyle of the elderly or the fact that some of them were bedridden patients, which caused the caretaker to let the abnormality go unnoticed.

The most common symptom of unrecognized cardiac arrest was unconsciousness, which was the same as the study of Crabb et al. [12]. Unconsciousness was also the most reported symptom of cardiac arrest in our study as in the study of Watkins et al. and a recent American Heart Association adult basic and advanced life support guideline [1], [3]. By adding a recognition of breathing patterns such as absent or agonal or abnormal breathing, we may achieve a higher recognition rate of cardiac arrest. In the study of Watkins et al. determining breathing pattern can lead to an increase of 7 points of recognized cardiac arrest if the call handler was strict on the protocol [3]. The main obstacle of recognizing OHCA was the difficulty of determining the patient's respiratory status for bystanders [12]. Another important reported symptom of cardiac arrest in our study was seizure-like activity, which may mislead the call handler from recognizing cardiac arrest to epilepsy as in the study of Vaillancourt et al. [5], [13].

Third, we found a very low survival to hospital discharge rate $(6.3 \%)$ in the recognized cardiac arrest group (overall survival to hospital discharge rate was $3.4 \%$ ). Compared with the OHCA survival to hospital discharge rate of $10.6 \%$ in the same hospital in the previous 5 years [7]. This might be a result of the method of our data collection. In Thailand, especially in the Northeast region of Thailand, people believe that if someone will die soon, their relatives should bring them home, to die at home. So, we defined the people whose relatives brought the patient to die at home as a nonsurvivor, where the previous study may have defined those cases as survivor. The other possible reason for the lower survival rate might be the increased popularity of palliative care in Thailand that lets the patients looking at poor outcomes die peacefully instead of suffering. When compared to the recent global survival to hospital discharge rate of $8.8 \%$ [2], our study has also shown a very low survival to discharge rate. The main reasons are the lower rate of bystander CPR administered and the fact that there was no AED used in this study which were both key elements of survival of the American Heart Association adult basic and advanced life support guidelines [1].
To the best of our knowledge, this is the first study in Thailand to identify the recognition rate of cardiac arrest by EMS call handlers. Our study also reported the common symptoms of unrecognized $\mathrm{OHCA}$ in Thailand. However, there are some potential limitations to this study. First, due to the retrospective nature of the data collection, some data was missing. Second, this study is a single-center study that may not represent the entire country.

\section{Conclusions}

Unconsciousness is the most common symptom of unrecognized OHCA by the EMS in Thailand. Basic life support, especially the immediate identification of the patient's respiratory status should be considered a fundamental part of health education, taught in schools or public services to the Thai population.

\section{Acknowledgements}

The present study received funding from the Khon Kaen University Faculty of Medicine (RR64301). The authors would like to thank the physicians and nurses at the Srinagarind Hospital Accident and Emergency Department, and Khon Kaen University Faculty of Medicine's Department of Emergency Medicine for their support. The authors would like to thank Josh Macknick for acting as an English consultant.

\section{References}

1. Panchal AR, Bartos JA, Cabañas JG, Donnino MW, Drennan IR, Hirsch KG, et al. Part 3: Adult basic and advanced life support: 2020 American heart association guidelines for cardiopulmonary resuscitation and emergency cardiovascular care. Circulation. 2020;142(16):S366-468. https://doi. org/10.1161/cir.0000000000000918

PMid:33081529

2. Yan S, Gan Y, Jiang N, Wang R, Chen Y, Luo Z, et al. The global survival rate among adult out-of-hospital cardiac arrest patients who received cardiopulmonary resuscitation: A systematic review and meta-analysis. Crit Care. 2020;24(1):61. https://doi. org/10.1186/s13054-020-2773-2

PMid:32087741

3. Watkins $\mathrm{CL}$, Jones SP, Hurley MA, Benedetto V, Price $\mathrm{Cl}$, Sutton CJ, et al. Predictors of recognition of out of hospital cardiac arrest by emergency medical services call handlers in England: A mixed methods diagnostic accuracy study. Scand J Trauma Resusc Emerg Med. 2021;29(1):7. https://doi. 
org/10.1186/s13049-020-00823-9

PMid:33407699

4. National Institute for Emergency Medicine. Emergency Medical Triage Protocol and Criteria Based Dispatch; 2013. Available from: https://www.niems.go.th/1/Ebook/Detail/272?group=21.

5. Vaillancourt C, Charette ML, Bohm K, Dunford J, Castrén M. In out-of-hospital cardiac arrest patients, does the description of any specific symptoms to the emergency medical dispatcher improve the accuracy of the diagnosis of cardiac arrest: A systematic review of the literature. Resuscitation. 2011;82(12):1483-9. https://doi.org/10.1016/j.resuscitation.2011.05.020

PMid:21704442

6. Viereck S, Møller TP, Rothman JP, Folke F, Lippert FK Recognition of out-of-hospital cardiac arrest during emergency calls-a systematic review of observational studies. Scand J Trauma Resusc Emerg Med. 2017;25(1):9. https://doi. org/10.1186/s13049-017-0350-8

PMid:28143588

7. Buranasakda $M$, Wangsri $K$, lenghong $K$, Kotruchin $P$, Rattanasriha W. Survival outcome of out-of-hospital cardiac arrest in emergency medical service with and without physician staffed. Srinagarind Med J. 2017;32:105-10.

8. Monsomboon A, Chantawatsharakorn $P$, Suksuriyayothin $S$, Keorochana K, Mukda A, Prapruetkit N, et al. Prevalence of emergency medical service utilisation in patients with out-ofhospital cardiac arrest in Thailand. Emerg Med J. 2016;33:213-7. https://doi.org/10.1136/emermed-2015-204818

PMid:26531862
9. Lindner TW, Søreide E, Nilsen OB, Torunn MW, Lossius HM. Good outcome in every fourth resuscitation attempt is achievable--an Utstein template report from the Stavanger region. Resuscitation. 2011;82(12):1508-13. https://doi. org/10.1016/j.resuscitation.2011.06.016

PMid:21752524

10. Cairns KJ, Hamilton AJ, Marshall AH, Moore MJ, Adgey AA, Kee $\mathrm{F}$. The obstacles to maximising the impact of public access defibrillation: An assessment of the dispatch mechanism for outof-hospital cardiac arrest. Heart. 2008;94(3):349-53. https://doi. org/10.1136/hrt.2006.109785 PMid: 17540690

11. Lee SY, Ro YS, Shin SD, Song KJ, Hong KJ, Park JH, et al. Recognition of out-of-hospital cardiac arrest during emergency calls and public awareness of cardiopulmonary resuscitation in communities: A multilevel analysis. Resuscitation. 2018;128:106-11. https://doi.org/10.1016/j. resuscitation.2018.05.008

PMid:29746984

12. Crabb D, Elmelige $\mathrm{Y}$, Gibson Z, Ralston D, Harrell C, Cohen S, et al. 1451: Unrecognized cardiac arrests: A oneyear review of audio from emergency medical dispatch calls. Crit Care Med. 2020;48:702. https://doi.org/10.1097/01. ccm.0000645716.61959.85

13. Schwarzkoph M, Yin L, Hergert L, Drucker C, Counts CR, Eisenberg M. Seizure-like presentation in OHCA creates barriers to dispatch recognition of cardiac arrest. Resuscitation. 2020;156:230-6. https://doi.org/10.1016/j. resuscitation.2020.06.036 